Journal of The Magnetics Society of Japan Vol. 13, Supplement, No. S1 (1989)

(C) 1989 by The Magnetics Society of Japan

\title{
BIT OBSERVATION ON PERPENDICULAR RECORDING MEDIA USING MAGNETO-OPTICAL MICROSCOPY
}

\author{
K. FUKUZAWA, J. KISHIGAMI and Y. KOSHIMOTO \\ NTT Applied Electronics Laboratories \\ 3-9-11, Midori-cho, Musashino-shi, Tokyo 180, Japan
}

\begin{abstract}
Magneto-optical microscopy using digital image processing is applied to observation of magnetization bit patterns in double-layer media. The bit patterns are found to depend on pole shapes and magnetomotive force. A reversal domain is observed on both sides of a track if the leading pole of the head is wider than the trailing one. The reason is that magnetization bits are written not by the longitudinal component but by the vertical component of head field. Moreover, the patterns become obscure with increasing magnetomotive force because of the saturation of media underlayers. Thin film heads with double-layer media can perform adequately in perpendicular recording by designing pole shapes and setting the magnetomotive force.
\end{abstract}

\section{INTRODUCTION}

To realize perpendicular recording in rigid disk drives, various heads are presented[1,2]. Thin film heads combined with double-layer media are valuable because they have a high record and reproduction efficiency. Head fields of this combination have strong longitudinal components, and the write process is complicated. That is why the write process of these combinations has not been properly clarified.

Written bit observation is important for investigation of the write process. Bits on doublelayer media has been observed by Bitter solution techniques. By this method magnetization can not be observed, but the the magnetization gradient can. Magneto-optical microscopy is useful for direct observation of magnetization. However, the Kerr rotation angle for $\mathrm{Co}-\mathrm{Cr}$ film is so small that there are few reports on bit observation by this method[2]. Recently digital image processing has been applied to this method for improving weak contrasts[4-6].
We have applied this method to the bit observation of $\mathrm{Co}-\mathrm{Cr}$ films and investigate the write process for a thin film head combined with double-layer media. The magnetomotive force dependency of written bits is also described.

\section{APPARATUS}

A modified polarized microscope (Nikon Microphot-fx) with a mercury arc (100 W) light source is used. The sample is obliquely illuminated to observe both perpendicular and longitudinal magnetization. The extinction ratio has been improved by use of a Glan-Thomson prism as the polarizer, and selecting objective lens. Camera signals are fed to an image processor (Nippon avionics Excel). The signals are averaged, and a difference image between opposite polarizer settings is acquired. By this method, magneto-optical contrasts can be enhanced and nonmagneto-optical contrasts can be reduced[7]. Finally, the required gray level of image is expanded to improve contrast. 


\section{RESULTS AND DISCUSSION}

\section{Magnetization bit patterns}

Figure 1 shows a magnetization bit patterns image. The media is composed of a $\mathrm{Co}-\mathrm{Cr}$ film (thickness $0.3 \mu \mathrm{m}$ ) and a Co-Zr film (thickness $1.0 \mu \mathrm{m})$. Head $\# 1$ of Table 1 is used in the experiment. Characteristic patterns are seen. They are different from bit patterns written in longitudinal media by the same type heads. Reversal domains on both sides of tracks are observed. The patterns are schematically shown in Figure 2. In Figure 2, length $l a$ is nearly equal to the width of the leading pole of head, and $l b$ is almost equal to that of the trailing pole. Magnetic transition of the reversal domain is located in different place from that of the domain in the center of the track. Transition difference is almost equal to the sum of the gap and the trailing pole lengths. This result implies that magnetization bits are written by the field of the backward edge of the trailing and the leading poles.

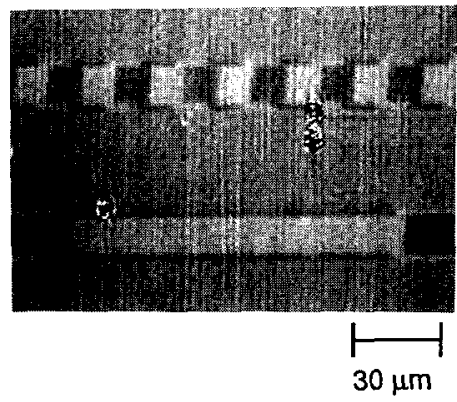

Fig. 1 Observed bit patterns.
Table 1. Head specifications

\begin{tabular}{lccc}
\hline Head & $\begin{array}{c}\text { Track width of } \\
\text { leading Pole } \\
(\mu \mathrm{m})\end{array}$ & $\begin{array}{c}\text { Track width of } \\
\text { trailing Pole } \\
(\mu \mathrm{m})\end{array}$ & Gap length \\
\hline$\# 1$ & 16 & 11 & $(\mu \mathrm{m})$ \\
$\# 2$ & 8 & 8 & 0.6 \\
\hline
\end{tabular}

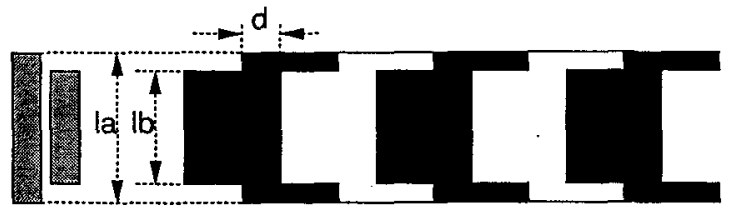

Poles

Fig. 2 Schematic magnetization bit patterns and poles. Black regions and white regions represent opposite domains. Head runs to the left direction.

Figure 3 shows calculations for the head field combined with the double- layer media. Computation is performed by a three dimensional integral equation method. Only the permeability of the soft magnetic underlayer is taken into account as magnetic characteristics of the media. In comparison with bulk $\mathrm{Co}-\mathrm{Zr}$, the permeability is small because the underlayer has no anisotropy. In Figure 3, (a) and (b) shows vertical component distribution on line $a-a^{\prime}$ and $b-b^{\prime}$, respectively. The leading pole is the wider one. Assuming that the coercivity of $\mathrm{Co}-\mathrm{Cr}$ films is $1000 \mathrm{Oe}$, magnetic transition occurs at $x=0.1 \mu \mathrm{m}$ in (a) and at $x=3.6 \mu \mathrm{m}$ in (b). Points $x=0.1 \mu \mathrm{m}$ and $x=3.6 \mu \mathrm{m}$ correspond to the backward edges of the leading and trailing poles, respectively. The calculation results support the fact that magnetization bits are written by the field edge of the each of the poles. 

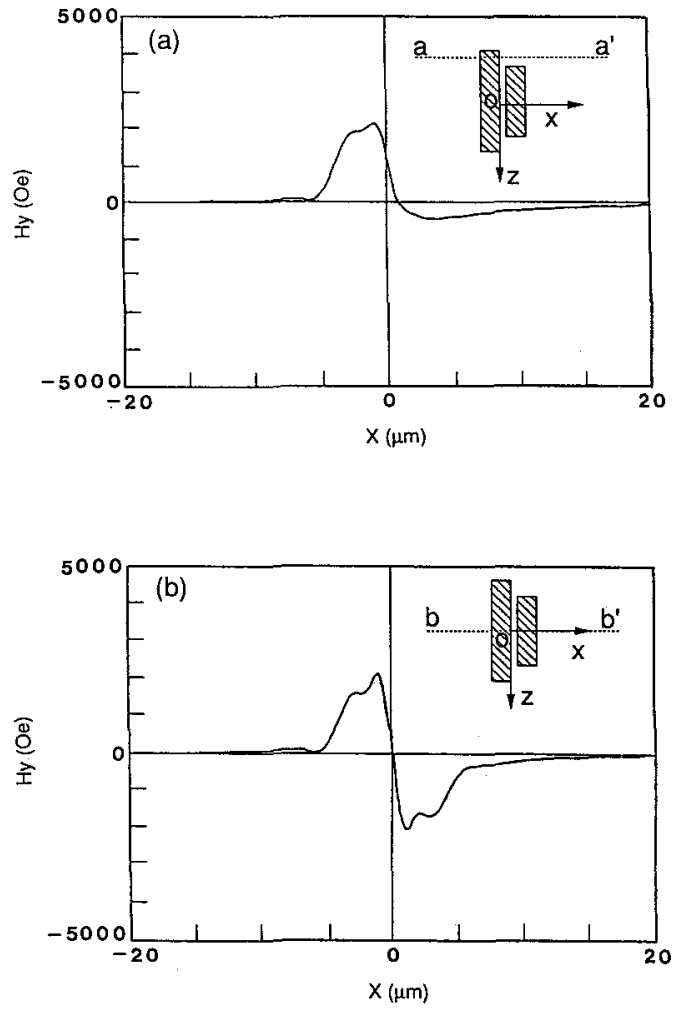

Fig. 3 Caluculated vertical component for the head field. Point $O$ represents origin. Head permeability and soft magnetic underlayer are 2000 and 400 , respectively. Pole configuration of corresponds to head \#1 in Table1. PI is $3.0 \mu \mathrm{m}$ for both poles, $2 \mathrm{~g}$ is $0.6 \mu \mathrm{m}$.

(a) Hy on the line a-a' .

(b) Hy on the line b-b'.

This is derived from bit observation and field calculations that the write process of thin film heads combined with double-layer media is similar to that of heads with a pair of single poles with double-layer media. Figure $4($ a) shows a magnetization bit image written by a head with misaligned poles at the other edge. Pole shape is schematically shown in Figure 4(b). The bit pattern corresponding to the pole configuration is observed. In this combination of head and media, track width is not equal to the gap but to the width of the wider pole. It is very different from longitudinal recording.

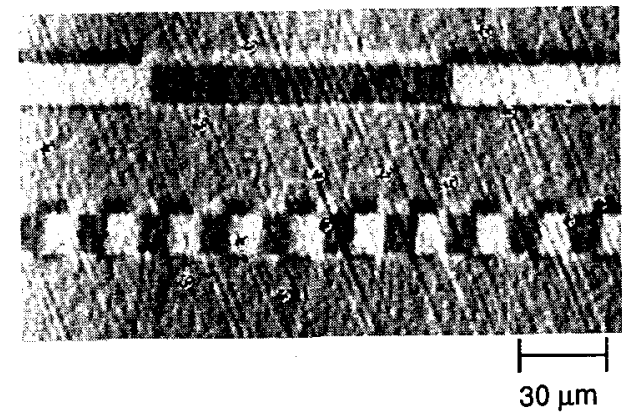

(a)

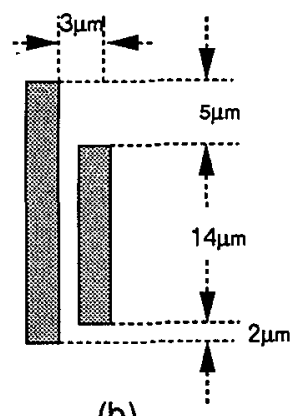

(b)

Fig.4 Bit patterns written by a misaligned head.

(a) Observed bit patterns.

(b) Schematic configuration for poles of the misaligned head. Left pole and right pole are leading and trailing poles, respectively.

\section{Magnetomotive force dependence}

Figure 5 shows observed bit patterns for increasing magnetomotive force. The recording head configuration is shown as \#2 of Table 1 . Media is the same as used in the former experiment. Figure 6 shows readback voltage vs. magnetomotive force. At a magnetomotive force of $\mathrm{NI}=0.34 \mathrm{AT}$, both readback voltage and contrast 
of bit image is maximum and magnetic transition is clearly observed. By increasing magnetomotive force to more than $0.34 \mathrm{AT}$, image contrast decreases and transition becomes more obscure. Readback voltage also becomes lower, corresponding to degradation of the written bits.

Figure 7 shows calculated head fields vs. magnetomotive force. According to Figure 7, head fields include more longitudinal components on increasing the magnetomotive force. The reason is because the soft magnetic underlayer is saturated. In this region, not only the vertical component, but also the longitudinal component affects the write process. To analyze this write process, a vector magnetization process for $\mathrm{Co}$ Cr film should be accounted for.

(a)

(b)

(c)

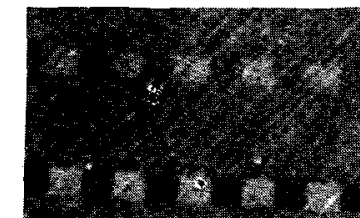

(d)

(e)

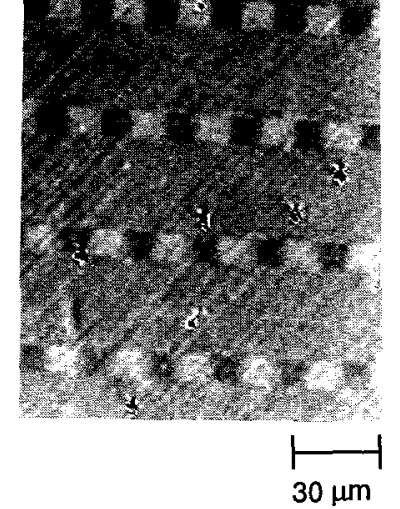

Fig. 5 Observed bit patterns on increasing magnetomotive force.
(a) $\mathrm{NI}=0.17 \mathrm{AT}$, (b) $\mathrm{Nl}=0.34 \mathrm{AT}$,
(c) $\mathrm{Nl}=1.70 \mathrm{AT}$, (d) $\mathrm{Nl}=3.40 \mathrm{AT}$,
(e) $\mathrm{NI}=5.10 \mathrm{AT}$.

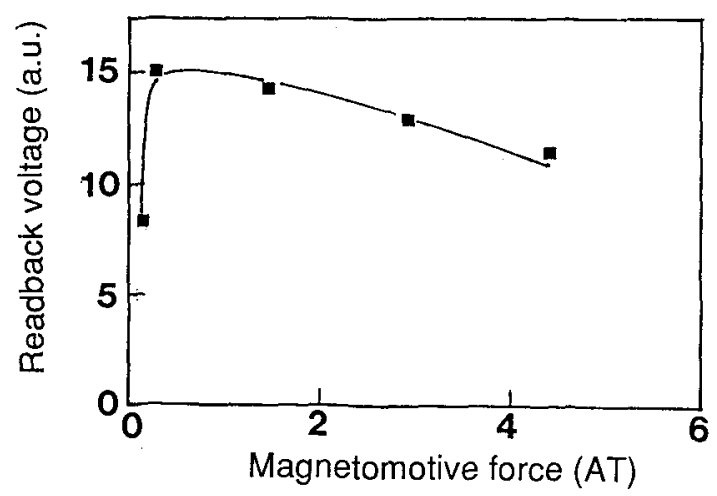

Fig. 6 Readback voltage versus magnetomotive force.

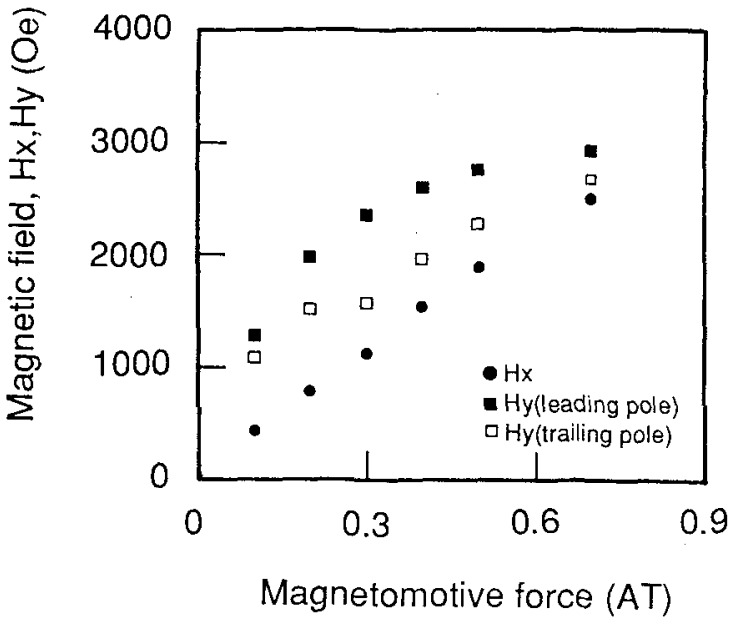

Fig. 7 Calculated head field versus magnetomotive force. Head fields are peak value. 


\section{CONCLUSION}

Magneto-optical microscopy with digital image processing was applied to the observation of magnetization bits in double-layer media. The bit patterns were found to depend on pole shapes and magnetomotive force. The write process of a thin film head combined with double-layer media is similar to that of a pair of single-pole heads with double-layer media. Magnetization bits are written by the vertical field of the backward edge of each pole. Therefore, when the leading pole is wider than trailing one, the reversal domain which is not overwritten by the trailing pole remains on both sides of the track. The magnetic transition position of the reversal domain is different from that of the domain in the center of the track. Increase in magnetomotive force degrades bit patterns and reduce readback voltage, because the head field includes a large Iongitudinal component caused by saturation of the soft magnetic underlayer and the write process is affected by the longitudinal component. By designing the pole configuration and setting the magnetomotive force, thin film heads with double-layer media can perform adequately in perpendicular recording.

\section{ACKNOWLEDGEMENT}

The authors are indebted to Dr. Akio Tago and Dr. Shigeru Hirono for sample preparation. They are also pleased to acknowledge to Dr. Shigehisa Fukui and Toru Takeda for their contributing discussions.

\section{REFERENCES}

[1] K. Kiuchi, H. Wakamatsu, F. Suzuki and H. Takagi, IEEE Trans. Magn., "High-energy Co$\mathrm{Cr}$ thin films sputtered on glass disks for perpendicular recording" Vol. 24 NO. 6, pp.2341-2343, November 1988.
[2] H. Schewe, IEEE Trans. Magn., "A new $\mathrm{read} /$ write concept for vertical recording" Vol. 24, NO. 6, pp.2410-2412, November 1988.

[3] F. Schmidt and A. Hubert, J. Magn. Magn. Mat., "Domain observations on CoCr-layers with digitally enhanced Kerr-microscope" Vol. 61, pp. 307-320, 1986.

[4] K. Shirae and K. Sugiyama, J. Appl. Phys., "A CCD image sensor and a microcomputer make magnetic domain observation clear and convenient" Vol. 53, NO. 11, pp. 8380-8382, November 1982.

[5] F. Schmidt, W. Rave and A. Hubert, IEEE Trans. Magn., "Enhancement of magneto-optical domain observation by digital image processing" Vol.MAG- 21, NO. 5, pp. 1596-1598, September, 1985.

[6] D. Herman Jr. and B. E. Argyle, IEEE Trans. Magn., "Magneto-optic observation of domain motion $\mathrm{s}$ and instabilities in small permalloy elements" Vol. MAG-22, NO. 5, pp. 772-774, September 1986.

[7] D. Treves J. App. Phys. "Magneto-optic detection of high-density recordings" Vol. 38, NO. 3, pp. 1192-1196, March 1967. 